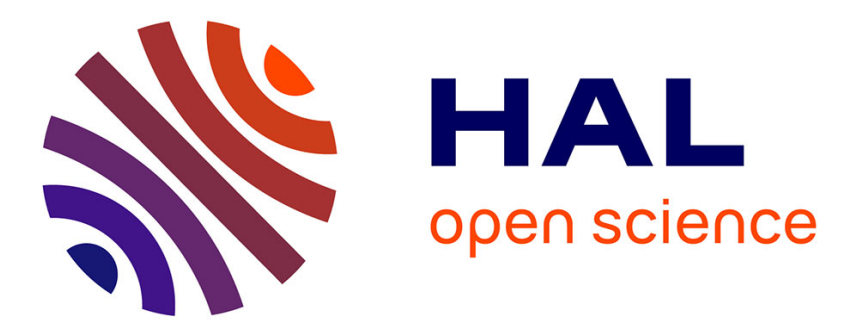

\title{
L'ONU contre la famille? La famille dans les textes onusiens depuis la Déclaration universelle des droits de l'homme de 1948
}

\author{
Blandine Chelini-Pont
}

\section{- To cite this version:}

Blandine Chelini-Pont. L'ONU contre la famille? La famille dans les textes onusiens depuis la Déclaration universelle des droits de l'homme de 1948. Recherches et prévisions, 1999, 10.3406/caf.1999.1849 . hal-02516384

\section{HAL Id: hal-02516384 \\ https://hal-amu.archives-ouvertes.fr/hal-02516384}

Submitted on 23 Mar 2020

HAL is a multi-disciplinary open access archive for the deposit and dissemination of scientific research documents, whether they are published or not. The documents may come from teaching and research institutions in France or abroad, or from public or private research centers.
L'archive ouverte pluridisciplinaire HAL, est destinée au dépôt et à la diffusion de documents scientifiques de niveau recherche, publiés ou non, émanant des établissements d'enseignement et de recherche français ou étrangers, des laboratoires publics ou privés.

\section{(ㅇ)(1) $\$$}

Distributed under a Creative Commons Attribution - NonCommercial - NoDerivatives| 4.0 


\title{
L'ONU contre la famille? La famille dans les textes onusiens depuis la Déclaration universelle des droits de l'homme de 1948
}

In: Recherches et prévisions, N. 56, juin 1999. pp. 21-31.

\begin{abstract}
Résumé
La famille est une réalité que la Déclaration universelle des droits de l'homme de 1948 ne reconnaît que par détour et qu'elle ne définit pas. Elle sert de repoussoir, entre les années cinquante et quatre-vingt, dans la lutte titanesque de l'ONU pour la promotion de la dignité et de la liberté de la personne, conçue hors de ses attaches naturelles. Cependant, malgré la tonalité majoritairement négative qui se dégage des textes concernant la famille, on peut noter une forme de prise de conscience. $\mathrm{A}$ l'attitude suspicieuse succède, au début de la décennie quatre-vingt, une tentative soutenue de défense de la famille comme un espace privilégié de développement personnel. Cette tentative ne paraît pas avoir réussi et l'ONU, portée à protéger les faibles dominés dans la structure familiale, a accumulé beaucoup de retard en matière de promotion positive d'un « droit à la famille " comme nouveau droit de la personne à intégrer aux autres droits, ce qui fragilise sa légitimité.
\end{abstract}

Citer ce document / Cite this document :

Chélini-Pont Blandine. L'ONU contre la famille ? La famille dans les textes onusiens depuis la Déclaration universelle des droits de l'homme de 1948. In: Recherches et prévisions, N. 56, juin 1999. pp. 21-31.

doi : 10.3406/caf.1999.1849

http://www.persee.fr/web/revues/home/prescript/article/caf_1149-1590_1999_num_56_1_1849 


\title{
L'ONU contre la famille?
}

\author{
La famille dans les textes onusiens depuis la Déclaration \\ universelle des droits de l'homme de 1948
}

\author{
Blandine Chélini-Pont *
}

La famille est une réalité que la Déclaration universelle des droits de l'homme de 1948 ne reconnaît que par détour et qu'elle ne définit pas. Elle sert de repoussoir, entre les années cinquante et quatre-vingt, dans la lutte titanesque de l'ONU pour la promotion de la dignité et de la liberté de la personne, conçue hors de ses attaches naturelles. Cependant, malgré la tonalité majoritairement négative qui se dégage des textes concernant la famille, on peut noter une forme de prise de conscience. A l'attitude suspicieuse succède, au début de la décennie quatrevingt, une tentative soutenue de défense de la famille comme un espace privilégié de développement personnel. Cette tentative ne paraît pas avoir réussi et l'ONU, portée à protéger les faibles dominés dans la structure familiale, a accumulé beaucoup de retard en matière de promotion positive d'un "droit à la famille" comme nouveau droit de la personne à intégrer aux autres droits, ce qui fragilise sa légitimité.

- Docteur en Histoire, professeur agrégée - université d'Aix-Marseille. omment le développement réel des droits de l'homme arrive-t-il à intégrer la réalité familiale ? Cette question est contradictoire parce que la Déclaration universelle et toute la philosophie juridique qui en a découlé depuis s'intéressent à la personne singulière, dans une synthèse difficile entre l'individualisme libéral des Lumières et le personnalisme chrétien, l'un axé sur la liberté de l'homme, l'autre sur sa dignité. Cette synthèse ne masque pas leurs divergences très profondes sur la définition de l'être humain, mais elle a eu le mérite de présenter une formule universelle accessible et cohérente. Toutefois, cette synthèse philosophique et juridi- que laisse en suspens la question de la famille, qui subordonne le singulier à sa propre logique communautaire.

Partant du texte fondateur de la Déclaration universelle, il faut chercher des réponses dans les textes adoptés par l'Assemblée générale des Nations unies relatifs aux droits de l'homme. Le corpus est considérable, entre les déclarations, principes, directives, normes et recommandations qui n'ont pas de force obligatoire, mais une force morale indiscutable, et les pactes, protocoles et conventions qui lient juridiquement les Etats qui les ratifient (1). Depuis 1994, l'information, plus fragmentaire, se trouve dans les 
comptes rendus des grandes conférences internationales, comme celle de 1993 sur les droits de l'homme, ayant fait l'objet d'une déclaration dite de Vienne, ainsi que dans des rapports d'experts qui servent d'outil de réflexion, soit pour orienter les objectifs à long terme de l'ONU, soit pour légitimer ses programmes d'action.

\section{La famille, une réalité contraignante}

Avec ce matériel abondant, on constate que la famille est reconnue comme "l'élément naturel et fondamental de la société et (qu'elle) a droit à la protection de la société et de l'Etat ". Mais, ce postulat présenté, elle est vite considérée comme une réalité contraignante contraire à l'épanouissement de l'être humain. Elle est regardée comme le véhicule privilégié de la "tradition". La famille entérinerait la discrimination et la violence à l'égard des faibles, essentiellement des femmes et des enfants, et ce quelles que soient ses formes dans les sociétés humaines. Jusqu'aux années quatre-vingt, telle est la tonalité générale des grands textes onusiens.

Cependant, à la fin des années quatre-vingt, une inflexion nouvelle émerge sous l'officialisation de la lutte contre la discrimination sexuelle et la violence dans les familles, marquée par des réflexions sur la place de la famille dans la lutte contre la pauvreté, sur son rôle de dynamique économique et d'espace affectif incomparable qu'il convient de préserver et de privilégier (2). Cette tendance se concrétise par une année entière de promotion en 1994.

Où en est-on aujourd'hui ? Après 1994, Année internationale de la famille sous l'égide de l'ONU, cette inflexion est loin de dominer, et nous sommes dans une situation paradoxale où les propositions faites, les recherches accomplies pour renforcer la famille se sont multipliées, sans aboutir à de vrais résultats parce que l'ONU n'a pas fait le choix de cette direction. Prise dans la tourmente de la crise de la famille en Occident, elle hésite toujours à considérer l'éclatement familial généralisé comme la preuve du progrès des droits del'homme (3) contre la tradition, et la famille comme un lieu suspect, ainsi que le sous-entend le rapport de la Conférence de Pékin sur les femmes de 1995. L'année précédente, pourtant, dans le rapport de la Conférence internationale sur la population et le développement du Caire, un paragraphe entier avait été rédigé en faveur d'un appui socioéconomique envers la famille.

C'est dans un texte relativement récent, la Convention internationale sur la protection des droits des travailleurs migrants et de leur famille du 18 décembre 1990, que l'on trouve une définition objective et large de la famille : "l'expression "membres de la famille" désigne les personnes mariées aux travailleurs migrants ou ayant avec ceux-ci des relations qui, en vertu de la loi applicable, produisent des effets équivalents au mariage, ainsi que leurs enfants à charge et autres personnes à charge, qui sont reconnues comme membres de la famille en vertu de la législation applicable ou d'accords bilatéraux ou multilatéraux applicables entre les Etats intéressés".

\section{Se marier et fonder une famille : un droit fondamental}

Il faut comprendre ce long retard par la genèse des textes onusiens. Le trait le plus frappant de la Déclaration universelle est que l'article 16, concernant la famille, s'arrête surtout sur le droit de se marier et de fonder une famille comme droit fondamental à partir du moment où le mariage est conclu " avec le libre et plein consentement des futurs époux" (paragraphe 2), que le mariage est le fait " $d^{\prime}$ 'un homme et d'une femme nubiles sans aucune restriction quant à la race, la nationalité ou la religion" et qu'il ouvre $\grave{a}$ "des droits égaux durant le mariage et lors de sa dissolution » (paragraphe 1). Ces mêmes principes sont repris dans l'article 23 du Pacte international relatif aux droits civils et politiques (et dans l'article 12 de la Convention européenne des droits de l'homme). Avoir des enfants est également défini comme une liberté personnelle, un choix second du couple, qui découle de leur 
consentement et non d'un devoir de procréation inhérent à la fonction sociale du mariage, de sorte que les premiers textes n'insistent pas sur les devoirs conjugaux mutuels ni les devoirs parentaux car leur perspective est libérale.

\section{Une lutte contre les sociétés traditionnelles}

L'ONU a donc voulu protéger la liberté personnelle de se marier, ainsi que l'égalité juridique et le consentement des contractants comme base du lien conjugal a priori monogamique (4), sans s'intéresser à la famille comme lieu de vie des personnes. Ce faisant, l'ONU a engagé une lutte difficile pour imposer une structure juridique matrimoniale, avec consentement comme fondement du lien monogamique, égalité juridique, dissolution possible et liberté de l'enfantement. Elle a mené sa lutte contre les sociétés traditionnelles largement majoritaires dans le monde, dans lesquelles le mariage est une institution codifiée et très contraignante où le consentement personnel n'existe pas. Elle a également mené ce combat contre les législations occidentales quand celles-ci n'y correspondaient pas encore, par exemple sur l'autorité parentale conjointe et le divorce par consentement mutuel. L'évolution comparée des droits matrimoniaux atteste des larges progrès de ce modèle onusien en quarante ans.

Le combat onusien pour l'application des Droits de l'homme aux femmes a été, dès le départ, un combat ouvert contre les types de famille autres que celle définie plus haut. Ainsi, une convention spéciale a été élaborée sur le consentement au mariage, l'âge minimum du mariage, dont la précocité coutumière est souvent la règle, et l'enregistrement des mariages (5), afin de les rendre obligatoires dans les droits nationaux. Dans son préambule, cette convention rappelle que certaines coutumes, anciennes lois et pratiques intéressant le mariage et la famille sont incompatibles avec les principes énoncés dans la Charte et la Déclaration universelle. En conséquence, tous les Etats doivent prendre des mesures pour les abolir, établir des sanctions en cas de non-respect et créer un service de l'état civil, première étape pour légaliser les mariages et les contrôler.

Plus précise, la recommandation $\mathrm{du}$ $1^{\text {er }}$ novembre 1965 sur le même thème (6) introduit l'idée que les pratiques matrimoniales traditionnelles sont une des formes de l'esclavage dénoncé par la Convention de 1956, quand ne sont pas respectés le consentement, l'enregistrement et l'âge nubile de la mariée (7).

Dans la très importante Déclaration sur l'élimination de la discrimination à l'égard des femmes (RAG 2263XXII du 7 novembre 1967), le cadre familial est également considéré comme le lieu de prédilection de leur souffrance. L'ONU s'est attaqué au statut inférieur ou à l'absence de statut des femmes dans la loi et dans les comportements. Elle dénonce les mentalités qui les façonnent (8).

\section{Pour l'égalité des droits}

La Déclaration de 1967 impose l'égalité des droits constitutionnels, l'égalité des droits civils (article 6), que la femme soit mariée ou non, l'accès égal à l'éducation et à l'activité économique. Les droits politiques et la participation des femmes à la vie publique ont fait l'objet d'une Convention particulière en 1952. L'égalité dans les lois successorales a fait l'objet d'une résolution du Conseil économique et social en 1962, en même temps que l'amélioration de la condition de la mère célibataire. Toutes ces mesures et d'autres non citées se retrouvent dans la Convention sur l'élimination de toutes les formes de discrimination à l'égard des femmes du 18 décembre 1979 (résolution de l'Assemblée générale 34/180). Cette Convention a fixé les objectifs de la décennie des Nations unies pour les femmes, parmi lesquelles le principe de conférences internationales de la femme, dont la première s'est tenue à Nairobi en juillet 1985.

Ces textes dessinent dans la répétition de leurs exigences un modèle familial préfé- 
rentiel, celui où la femme, respectée en tant que personne, jouit des mêmes capacité juridiques que l'homme alors que le mariage la conduit par le droit coutumier à une infériorité sur tous les plans (9). Cette insistance et la promotion de la femme comme bataille met la famille dans une posture "défensive " par rapport aux droits de la femme. L'ONU depuis les années quatrevingt $s^{\prime}$ est orientée dans des programmes d'actions de promotion de la femme, d'amélioration de sa situation économique et de sa participation au développement collectif, avec des conventions spécifiques comme la Convention sur les travailleurs ayant des responsabilités familiales de 1981 . Elle a privilégiél'autonomisation de la femme par le travail. Dans cette optique, la famille fait encore figure de lieu d'enfermement et de dépendance et le travail est présenté comme un moyen d'accès à la liberté.

De même, l'ONU a posé le principe que la promotion de la femme passait par la maîtrise de sa fécondité comme "élément important pour l'exercice de ses autres droits ". Les paragraphes 156 à $159 \mathrm{du}$ rapport de 1985 de la Conférence mondiale de la femme, paragraphes refusés par la délégation du Saint-Siège (seul refus homologué dans le texte), véhiculent ainsi l'idée que la fécondité " incontrôlée " des femmes nuit à leur liberté car elle est l'origine de la plupart des contraintes physiologiques et culturelles qui s'imposent à elles.

\section{Des coutumes néfastes à l'encontre des femmes}

La liste est longue des reproches directs que l'ONU a pu dresser contre les familles, notamment dans son rapport de 1985. Tenantes de la tradition dans laquelle les hommes sont tout-puissants, les familles pratiquent à l'encontre des femmes des coutumes néfastes comme l'excision, le mariage précoce sans consentement, parfois précédés d'arrangements financiers ou d'une vente effective, les tabous nutritionnels, les pratiques dangereuses entourant la naissance et la préférence marquée, par des rites particuliers, pour les enfants mâles.
A ces discriminations pratiquées dans les familles, l'ONU a rajouté la dénonciation de la violence contre les femmes en famille. Une résolution du Conseil économique et social du 24 mai 1984 en a fait l'objectif des recherches du service de la Promotion de la femme de Vienne et de sa réunion sur la violence dans les foyers en 1986. Le résultat de ce travail a été un document impressionnant, publié en 1989 sous le titre "Violence contre les femmes dans la famille", dont voici un extrait : "on peut affirmer que la violence est inscrite dans la nature de maintes situations familiales et que beaucoup de femmes sont assassinées, agressées, en particulier sexuellement, menacées et humiliées dans leur foyer par les hommes auxquels elles sont liées et que ce type de situation ne semble ni inhabituel ni exceptionnel».

\section{La protection des femmes contre la violence conjugale}

L'ordre social dans toutes les cultures tient les femmes en infériorité et la violence conjugale est la forme la plus visible de cette inégalité culturellement inculquée, car la famille est la "réplique fidèle et la confirmation de la société " (10). L'abondance des exemples de violence - surtout conjugale dans l'étude - est largement suffisante pour convaincre le lecteur que plus la société est traditionnelle plus cette violence est répandue. Pour autant, les pays occidentaux ne sont pas épargnés dans cette étude. En conclusion, les experts de cette recherche proposent un arsenal juridique et éducatif, notamment en faveur des administrations de protection (santé, police), afin de réduire la tolérance sociale à ce phénomène.

On retrouve en faveur des enfants, cette même lutte contre l'arbitraire familial, ennemi à abattre. L'enfant, objet de textes onusiens fondamentaux, est défini comme sujet du droit pour être soustrait à la violence familiale. Les textes sont pensés de manière à le protéger de l'influence néfaste que la famille peut exercer sur lui, par une éducation intolérante, négligente ou défaillante. L'absence ou le refus d'éducation intellectuelle, notamment pour les filles, le 
travail précoce pour con tribuer ou faire vivre réellement la famille sont par ailleurs violemment dénoncés.

Cette méfiance à l'égard du milieu familial est notable dans les textes qui parlent du droit à l'éducation et à l'enseignement. La famille est certes libre de ses choix éducatifs, mais ceux-ci sont sérieusement balisés par un enseignement scolaire obligatoire, de préférence public et gratuit. L'enseignement peut être privé pour des raisons confessionnelles, mais dans un cadre défini par la loi publique (11). Le principe sousentendu est que l'éducation des enfants doit être conforme aux principes de la charte de l'ONU, alors que l'espace éducatif de la famille est celui de la transmission des valeurs culturelles et de la religion d'origine des parents, clairement énoncés comme des droits (article 18 du Pacte international relatif aux droits civiques et politiques), sans que l'on sache exactement de quoi il $s^{\prime}$ agit, cette transmission étant surtout celle des valeurs traditionnelles qui sont combattues par l'ONU, comme source rétrograde $d^{\prime}$ 'injustice et d'arbitraire.

\section{Education des enfants : un droit de regard des Etats}

La Déclaration concernant la promotion parmi les jeunes des idéaux de paix, de respect mutuel et de compréhension entre les peuples (12) rappelle, dans son principe II, que " tousles moyens d'éducation, y compris étant donnéson importance capitale, l'éducation donnée par les parents ou la famille, doivent promouvoir parmi les jeunes les idéaux de paix, d'humanisme, de liberté et de solidarité internationale et doivent leur faire connaître le rôle confié à l'ONU en tant que moyen de préserver et de maintenir la paix et de favoriser la compréhension et la coopération internationale ". On ne peut qu'applaudir à ce programme, mais il laisse transparaître une évidence officiellement inavouée et politiquement incorrecte: le respect des conventions de l'ONU impose aux Etats un véritable droit de regard sur l'éducation de l'enfant. Le respect des valeurs familiales est subordonné à cet impératif, de même que le respect de la famille passe d'abord par l'application d'un modèle juridique précis.

Cependant, c'est à la faveur des textes onusiens qui touchent l'enfant et dans lesquels la charge dénonciatrice contre la famille pourrait être la plus forte, que la famille est paradoxalement présentée comme un espace à respecter, à protéger et à encourager. Les textes, là encore, donnent un matériel éclairant - quoique fragmentaire - sur l'aspect à la fois nécessaire et positif de la famille dans l'éducation de l'enfant.

\section{La famille : milieu de vie privilégié pour l'enfant}

La Déclaration des droits de l'enfant de 1959 privilégie particulièrement la famille comme lieu d'affection, de sécurité morale et matérielle (principe 6). La Convention sur les droits de l'enfant de 1989 rappelle, en préambule, que tous les Etats parties sont " convaincus que la famille, unité fondamentale de la société et milieu naturel pour la croissance et le bien-être de tous ses membres et en particulier des enfants, doit recevoir la protection et l'assistance dont elle a besoin pour pouvoir jouer pleinement son rôle dans la communauté ". Ils "reconnaissent que l'enfant, pour l'épanouissement harmonieux de sa personnalité, doit grandir dans le milieu familial, dans un climat de bonheur, d'amour et de compréhension ». L'article 7 reconnaît à l'enfant le droit, "dans la mesure du possible, de connaître ses parents et d'être élevé par eux". L'article 9 interdit que l'enfant soit séparé de ses parents contre leur gré, à moins de son intérêt supérieur dûment prescrit par la loi. Ce même article et l'article 10 posent le droit pour l'enfant d'entretenir, en cas de séparation volontaire ou involontaire de ses parents et d'avec ses parents, des relations personnelles régulières et directes (13).

Le milieu familial de l'enfant est systématiquement préféré et l'adoption comme solution de remplacement quand la famille naturelle fait défaut par défaillance, maltraitance ou absence, est strictement 
réglementée par l'article 21. Une Déclaration antérieure, du 3 décembre 1986, relative à l'adoption a, par ailleurs, posé le principe du placement de l'enfant en dernier ressort, dans le seul cas de son intérêt et de préférence dans une famille plutôt que dans une institution (14).

\section{La famille nucléaire, modèle du cadre juridique}

A travers ces textes, on mesure à quel point la famille nucléaire est le modèle inspirateur du cadre juridique imposé, ce qui peut réduire considérablement leur portée, puisque ce type de famille, certes en extension, particulièrement dans sa forme monoparentale en Occident, n'est pas majoritaire dans le monde. Mais même ainsi réduite, la famille est présentée positivement comme le milieu de vie épanouissant par excellence pour les enfants (15).

Cette préférence pour la famille, milieu de vie naturel épanouissant, se retrouve dans les textes qui touchent le respect des droits des personnes déficientes mentales. Par la Déclaration du 20 décembre 1971 et les Principes pour leur protection du 17 décembre 1991, les déficients mentaux ont le droit " de vivre au sein de (leur) famille ou d'un foyer s'y substituant et (de) participer à différentes formes de la vie communautaire" (16). Ils ont le droit "dans la mesure du possible, d'être traités et soignés dans le milieu où ils vivent". Le droit, "chaque fois que cela est possible quand ils sont soignés dans un service de santé mental, de suivre leur traitement à proximité de leur domicile ou du domicile des membres de leur famille ou d'amis, et de retourner dès que possible dans leur milieu de vie". Bref, les déficients mentaux ont droit à " un traitement adapté à leur milieu culturel » (17).

La préférence pour le milieu de vie "naturel " en cas de grande faiblesse se trouve également dans la Déclaration des droits des personnes handicapées de 1975 qui spécifie bien, dans son article 9, que le handicapé a droit de vivre au sein de sa famille ou d'un foyer s'y substituant où "le milieu et les conditions de vie doivent y être aussi proches que possible de ceux de la vie normale des personnes de son âge » (18).

La famille se dessine donc comme un véritable rempart affectif et la dernière Conférence mondiale sur les droits de l'homme, qui s'est tenue à Vienne en juin 1993, rappelle dans sa Déclaration que " pour que sa personnalité se développe pleinement et harmonieusement, l'enfant doit pouvoir grandir dans un environnement familial qui mérite, par conséquent, d'être plus largement protégé " (19). $L$ 'idée de la protection de la famille comme milieu de vie se fraye donc progressivement un chemin malgré tous les aspects liberticides de celle-ci, par la reconnaissance de son importance psycho-affective.

\section{Un véritable rempart affectif}

L'importance affective de la famille est moins visible dans les textes que sa fonction de ressource économique et de moyen de prospérité sociale reconnus assez tardivement par l'ONU. Avec quatre études fort complètes, publiées en 1987, apparaît un but nouveau et clairement affirmé : réduire l'approche jusque-là fragmentaire de la famille "qui risque de faire perdre de vue la famille comme entité à caractère global " (20). Plusieurs idées apparaissent. Tout d'abord, l'absence de famille comme phénomène émergeant fragilise émotionnellement et économiquement à la fois les personnes concernées et les sociétés dans leur ensemble. Eviter la rupture de la famille et prévenir cette rupture deviennent un objectif prioritaire. La deuxième idée est d'encourager, à côté des systèmes de protection sociale et d'allocations familiales, les programmes d'assistance aux familles qui sont actuellement incomplets et insuffisants dans leurs intentions.

Il s'agit de sortir de la logique d'assistance pour entrer dans une vraie politique de promotion de la famille, inventive et adaptée souplement aux situations locales et aux structures familiales. Parmi les mesures préconisées, des programmes de soutien psychologique et relationnel destinés aux 
familles pour éviter, préventivement ou pendant la crise, les ruptures familiales. $D^{\prime}$ autres programmes sont destinés aux enfants de parents célibataires ou divorcés, aux orphelins et aux enfants dénués de ressources ou de soutien. Des programmes de prévention et de réduction de la toxicomanie ou de l'alcoolisme dans les familles, des programmes de réinsertion spécifique pour les familles touchées par le chômage sont aussi proposés.

Les rapports ont tous la même conclusion. Les efforts accomplis par l'ONU touchant de manière séparée aux domaines de la population, de la protection sociale, du travail, du logement, de la sécurité sociale, de la santé et de la nutrition, du développement rural et de l'agriculture, de l'urbanisation, de la réforme agraire et de l'industrialisation, doivent être désormais intégrés ensemble dans l'idée de prendre en compte la réalité familiale comme une réalité universellement vécue.

\section{4 : Année internationale de la famille}

La défense de la famille par l'ONU s'est concrétisée en 1994 avec une Année internationale de la famille issue de la résolution de l'Assemblée générale 44/82 du 8 décembre 1989. Cette Année avait pour thème "les ressources et la responsabilité de la famille dans un monde en mutation ". Son but était de "sensibiliser davantage l'opinion auxquestions relatives à la famille " (21). Outre les thèmes onusiens classiques sur le renforcement de la législation sociale et salariale et la nécessité de l'assistance financière pour les familles en difficulté, les problématiques et objectifs prioritaires qui s'en dégagèrent ont été les suivantes : appui aux fonctions sociales de la famille (prise en charge matérielle, sanitaire, affective et éducative, transmission des valeurs et de la culture, socialisation et intégration sociale); appui à la fonction économique de la famille comme prestataire et consommatrice de services et agent de développement. Ces aspects sont repris dans les objectifs que se fixe le programme d'action de la Conférence sur la population et le développement tenue au Caire en 1994 (22) et de manière spécifique dans les buts et principes du rapport de la Conférence sur les établissements humains de 1996 (23).

Dans les derniers rapports annuels de l'ONU, rapport mondial sur le développement humain 1997 et rapport sur la situation sociale dans le monde 1997, des paragraphes entiers étudient la résistance des familles à la pauvreté par des stratégies collectives d'entraide et déplorent in fine que l'effritement de ces solidarités, sous l'effet de l'uniformisation des modes de vie, paupérise et fragilise davantage les personnes (24), même si ces mêmes rapports mettent en évidence l'incapacité des personnes à sortir de la pauvreté sous l'effet des contraintes familiales ici comprises comme l'effet d'une fécondité non maîtrisée (25). Reste que la famille est prise en compte dans les facteurs de bien-être économique et social et que ces rapports en exigent la protection comme moyen de lutter contre la paupérisation.

Le combat de l'ONU contre les aspects négatifs de la famille semble s'apaiser vers 1990. Son rôle positif émerge avec la nouvelle décennie et la critique devient plus constructive, se portant sur les méthodes efficaces pour optimiser ses fonctions affective, sociale et économique contre ses propres freins que sont pauvreté, chômage, violence, déséquilibre entre vie professionnelle et responsabilités familiales et négligence. En bref, la famille nouvelle version, agent de développement, de stabilité personnelle et collective, devient un droit de l'homme pour l'ONU.

A l'orée des années quatre-vingt-dix, l'ONU était prête à proposer un système cohérent de promotion de la famille comme milieu de vie de l'individu. Elle s'engageait à favoriser en quelque sorte un compromisentre le respect de la personne et l'aménagement de ses droits et de ses besoins dans la réalité familiale. On aurait pu croire que le principal problème allait être de concilier ou de réconcilier la diversité de la réalité familiale. C'est en tout cas ce que concluait un 
des rapports sur la famille de 1987 (26). Ce ne sont pourtant pas les difficultés d'une synthèse "universelle " devant la multitude des situations qui ont freiné cette tendance, mais la poursuite des combats déjà engagés par l'ONU en faveur du respect des droits de l'homme à l'intérieur de la famille.

\section{L'égalité des droits par l'égalité des sexes}

L'année 1994, décrétée Année de la famille, a paradoxalement rétabli l'ancienne direction. 157 Etats membres, 34 organismes et institutions spécialisées des Nations unies, plusieurs organismes intergouvernementaux et des centaines d'organisations non gouvernementales se sont mobilisés en faveur de l'égalité des droits par l'égalité des sexes. La dénonciation de l'inégalité des générations, des ressources, de la reconnaissance sociale, le refus de la violence au sein des familles, ont été l'objectif de la médiatisation onusienne, comme le précise très bien le rapport du secrétaire général à propos des résultats de l'Année de la famille de septembre 1995 (A/50/370).

Les tendances "offensives" contre la famille ont finalement été les plus visibles et les plus travaillées. La Conférence de Vienne sur les Droits de l'homme en 1993, la Conférence du Caire sur la population en 1994, celle d'Istanbul sur l'habitat humain en 1996 ont fait tourner toute leur problématique autour de l'idée que le développement durable passait par la traque de toutes les discriminations à l'égard des femmes et par la maîtrise technique de la reproduction humaine (27).

La Conférence mondiale sur les droits de l'homme de 1993, le Sommet mondial de Copenhague sur le développement social de mars 1995 et la Quatrième conférence mondiale sur les femmes à Pékin de 1995, insistent sur la protection spéciale des familles vulnérables, pauvres, qui ont souffert de la guerre, de la famine, ou de violence raciale ou ethnique, des familles monoparentales involontaires (veuvage, enfants naturels) ou volontaires (divorces, concubinage). Mais elles s'intéressent davantage à rappeler, et à avoir comme objectif, l'égalité entre les sexes et la promotion des femmes. L'injustice faite aux femmes du monde est amplement dénoncée.

L'élimination de la discrimination à l'égard des femmes (28), des petites filles, la mise en lumière du scandale éternel de la traite sexuelle des femmes et des filles (29), la condamnation des comportements violents en famille, toutes ces vérités renvoient la promotion de la famille à un futur éloigné. L'objectif important reste toujours la promotion de la femme par la maîtrise de sa fécondité, l'accès au travail, l'égalité juridique totale dans le droit civil, et surtout la reconnaissance sociale la plus large et son respect effectif dans les comportements et les mentalités collectives.

\section{Une déclaration universelle de la famille?}

Un texte sur la famille, pourquoi pas une convention ou une déclaration, permettrait de concilier la dénonciation des violations fréquentes des droits de la personne en famille, la proposition de normes juridiquesuniverselles pour y remédier, et l'énonciation positive d'articles spécifiques sur le groupe familial. L'absence d'une Convention internationale sur la famille est extrêmement grave. Elle contribue à affaiblir l'universalisme des droits de l'homme et à creuser l'écart entre l'Occident et le reste du monde sur la façon d'organiser et de gérer le lien familial. Un tel texte sur la famille encadrerait de manière universelle les conditions optimales de son fonctionnement et expliquerait les raisons de sa promotion, en affirmant, comme la Déclaration universelle des droits de l'homme a pu le faire en son temps, la transcendance de valeurs communes à tous les êtres humains, ce qui est le sens même et la justification de son existence (30). 
(1) Nous disposons, pour l'instant, d'un recueil d'instruments internationaux qui a été publié en 1994 par l'ONU dans le cadre des résolutions du $40^{\mathrm{e}}$ anniversaire de la Déclaration universelle des droits de l'homme de 1948. Ce recueil rassemble tous les textes adoptés jusqu'au 31 mars 1993. Autre recueil, celui des activités de l'ONU dans le domaine des droits de l'homme, publié en 1992 par le Centre pour les droits de l'homme de Genève. (2) «Les politiques visant à développer le potentiel des familles en tant que force vitale pour le développement sont peu nombreuses. Ceux qui élaborent ces politiques présument souvent que les familles qui ont besoin d'une assistance sont (...) déficientes... Les dirigeants doivent donc maintenant aborder la question de la famille en tant qu'élément central de politique. Dans ce contexte, ils devraient tenir compte du fait que a) les unités fondamentales pour la transmission de la vie sociale et des valeurs dans les sociétés ; b) les familles et les communautés dans lesquelles elles vivent sont interdépendantes ; c) les familles sont les unités dans lesquelles vivent généralement les individus et les intérêts des individus devraient donc être considérés dans ce contexte et $d$ ) les familles constituent la meilleure source d'appui affectif et matériel pour les enfants, les jeunes, les personnes âgées et les handicapés (...) La famille demeure l'organe de soutien le plus durable et le plus solide auquel peuvent recourir les individus ", Politiques nationales de la famille, NU, Vienne, La Famille, 1987, n³, pp. 48-50-56.

(3) «Certains des changements qui se sont produits ont porté sur la structure familiale: il y a eu passage de la famille élargie à la famille nucléaire et de la famille nucléaire à la famille monoparentale; les familles ont cessé d'être des unités de production à la suite de la migration d'une partie de leurs membres des zones rurales vers les zones urbaines; l'accent a été nis sur la primauté de l'individu; et il y a eu un net accroissement des taux de divorcialité. Ces changements font partie du processus de développement que chaque gouvernement s'efforce d'encourager, de promouvoir et de stimuler à titre de priorité absolueà l'échelle nationale ", Politiques nationales de la famille, NU, Vienne, La Famille, 1987, n³, p. 2.

(4) Voir Boucaud P., Le droit de se marier, in Revue trimestrielle des Droits de l'Homme, 1992, p. 1-46. L'auteur montre comment les pratiques du mariage dans le monde sont modifiées par ces normes désormais internationales, notamment le paragraphe sur la volonté des époux.

(5) 7 novembre 1962, RAG 1763 A(XVII).

(6) Résolution de l'Assemblée générale 2018.

(7) Toutes les occurrences relatives à la famille jusqu'aux années quatre-vingt concernent en fait la protection juridique des femmes dans le mariage : maintien de la nationalité de naissance de la femme mariée (Convention du 29 janvier 1957), régime égalitaire des biens, droit de la femme mariéeà exercer une profession indépendante (RCES 547 J XVIII, 12 juillet 1954), autorité parentale, tutelle et garde conjointe en cas de séparation (RCES 1207 XLII du 29 mai 1967). Ces idées sont reprises dans la RCES 1853 LVI du 16 mai 1974 sur la capacité juridique de la femme à exercer librement une profession, à administrer person- nellement ses revenus et les biens du ménage. Cette résolution exige également l'autorité parentale conjointe, l'égalité dans la dissolution du mariage et les effets juridiques de celle-ci.

(8) Article 3 : " toutes mesures appropriées doivent être prises pour éduquer l'opinion publique et inspirer dans tous les pays le désir d'abolir les préjugés et de supprimer toutes pratiques, coutumières et autres, qui sont fondées sur l'idée de l'infériorité de la femme". La Convention supplémentaire relative à l'abolition de l'esclavage (résolution du Conseil économique et social $608 \mathrm{XXI}$ ) parle de pratiques matrimoniales "comme institution et pratiques analogues à l'esclavage".

(9) Le rapport de la Conférence mondiale sur les femmes de 1985 insiste sur la nécessité pour tous les Etats d'adopter la Convention et demande l'abolition du droit coutumier, par le droit écrit et appliqué, l'égalité des femmes dans le droit du travail, la capacité juridique entière de la femme mariée, le droit de gérer, vendre et acheter librement dans le mariage, le droit de divorcer et de garder les enfants aux mêmes conditions quel que soit le sexe.

(10) "Une telle analyse situe la violence contre les femmes au sein du foyer dans la structure du mariage, de la famille et de la société en général, part en somme du principe qu'on ne peut dissocier les mauvais traitements dont sont victimes les femmes du contexte social et idéologique dans lequel ils se produisent. L'analyse conduit à mettre la famille en tant qu'inst itution mais aussi le rôle du corps social notamment des institutions ayant une mission d'assistance (police, tribunaux, corps médical), complices objectifs d'une struct ure qui accepte que l'homme fasse usage de sa violence pour maintenir sa domination sur la femme", "La violence contre les femmes dans la famille", service de la Promotion de la femme, Vienne, 1989, p. 33.

(11) Déclaration des droits de l'enfant, Résolution de l'Assemblée générale 1386 XIV du 20 novembre 1959, Convention relative aux droits de l'enfant, Résolution de l'Assemblée générale 44/ 25 du 20 novembre 1989, Déclaration sur les principes juridiques et sociaux applicables à la protection et au bien-être des enfants, envisagés sous l'angle des pratiques en matière d'adoption et de placement familial, Résolution de l'Assemblée générale 4185 du 3 décembre 1986, Convention concernant la lutte contre la discrimination dans le domaine de l'enseignement, Conférence des Nations unies, 14 décembre 1960.

(12) RAG 2037 XX du 7 décembre 1965.

(13) A ce propos, l'article 10 définissant le droit d'une famille (l'enfant et ses parents) de circuler librement fait écho à l'article 8 de la Convention internationale sur la protection des droits des travailleurs migrants et des membres de leur famille, Résolution de l'Assemblée générale 45/158 du 18 décembre 1990.

(14) Article 1: "Chaque Etat devrait donner la priorité au bienêtre de la famille et de l'enfant". Article 3 : " le bien-être de l'enfant est d'être élevé par ses parents naturels". Article 4 : "si l'enfant ne peut-être élevé par ses enfants naturels, ou si ceux-ci ne l'élèvent pas comme il convient, il faut envisager de le confier à des membres de la famille de ses parents, à une autre 
famille de remplacement - nourricière ou adoptive - ou, si nécessaire, à une institution appropriée ". Article 13: "le but premier de l'adoption est de procurer une famille permanente à l'enfant que ses parents naturels ne peuvent prendre en charge ".

(15) Dès les origines et avec constance, l'ONU a donc dirigé ses efforts à favoriser le développement d'un système de protection sociale, de prestations familiales, de suivi sanitaire, de protection spécifique de la maternité et de la petite enfance, dont on trouve la traduction la plus ancienne dans la Charte, et dans l'article 10 du Pacte international relatif aux droits économiques sociaux et culturels du 16 décembre 1966, puis dans toutes les déclarations et conventions spécifiques sur les femmes, et les enfants. (16) Article 4, Déclaration des droits du déficient mental, Résolution de l' Assemblée générale 2856 (XXVI) du 20 décembre 1971.

(17) Principe 7 des principes pour la protection des personnes atteintes de maladie mentale et pour l'amélioration des soins de santé mentale, Résolution de l'Assemblée générale 46/119 du 17 décembre 1991.

(18) Article 9 de la Déclaration des droits des personnes handicapées, Résolution de l'Assemblée générale du 9 décembre 1975, 3447 XXX. De même, une Assemblée mondiale sur le vieillissement s'est tenue les 26 juillet-2août 1982 à Vienne et son programme d'action, adopté par l'Assemblée générale en décembre de cette même année (Résolution de l'Assemblée générale 37/51, 3 décembre 1982), précise au chapitre VI que " la qualité de la vie n'est pas moins importante que la longévité et que, dans la mesure du possible, il faut permettre aux personnes âgées de mener, dans leurs propres familles, et leurs communautés, une vie où elles puissent connaître l'épanouissement personnel, la santé, la sécurité, la satisfaction et être appréciées, en tant que partie intégrante de la société" ".

(19) Rapport de la Conférence mondiale sur les droits de l'homme, 25 juin 1933, A/Conf/157/24, p. 26.

(20) Le Conseil économique et social, dans sa résolution 1983/ 23 de 1983 sur le rôle de la famille dans le processus de développement, a ainsi commandé une série d'études au Centre pour le développement social et les affaires humanitaire, à partir de thématiques communes et de rapports régionaux. La première publication est intitulée "Modèles à suivre pour assurer des services complets de protection de la famille et de l'enfance ". Elle poursuit des objectifs classiques en tentant de les rationaliser au maximum. La seconde est plus intéressante pour notre réflexion. Elle s'intitule "Le rôle de la famille dans le processus de développement ", direction reprise dans les deux dernières études sur "Les Politiques nationales de la famille : leur relation avec le rôle de la famille dans le processus de développement " et "Renforcer la famille : directives en vue de l'élaboration de programmes à cet effet ".

(21) Célébration de l'Année internationale de la famille, rapport du secrétaire général, $A G / 50 / 370$, 6 septembre 1995 .

(22) Rapport de la Conférence internationale du Caire, 5-
13 septembre 1994, A/Conf/171/13, Chapitre V sur la famille, pp. 32-35.

(23) Chapitre II, paragraphe V: "En ce qui concerne la planification des établissements humains, il faudrait tenir compte du rôle constructif de la famille dans leur conception, leur développement, leur gestion. La société devrait contribuer à créer, s'il y a lieu, toutes les conditions nécessaires à son intégration, à sa réunification, à sa préservation, à son amélioration et à sa protection, dans le contexte d'un logement convenable, et lui faciliter l'accès aux services de base et à des moyens d'existence durable ", rapport A/Conf/165/14, p. 21.

(24) « La famille est la principale source non officielle de sécurité, et souvent le seul moyen d'échapper à un dénuement extrême. Elle est particulièrement importante de par le soutien qu'elle apporte aux enfants, aux personnes âgées et aux handicapées. Partout, elle est aussi la source première de soutien pour les jeunes. Le jeune à la recherche d'un premier emploi compte davantage sur ses parents qu'il ne le ferait par la suite mais une famille dont les parents sont pauvres est incapable d'offirir à ses enfants les possibilités dont bénéficient les enfants de familles moins défavorisées. Al'échelle mondiale plus de la moitié des personnes âgées dépendent de leur famille pour subsister, et la famille demeure d'un grand secours pour les handicapés ", rapport sur la situation sociale dans le monde, ONU 1997, p. 155.

(25) « Du point de vue démographique, les pauvres présentent des caractéristiques quiles distinguent des autres groupes sociaux et compromettent leurs chances de développement. Les pauvres appartiennent souvent à des familles plus nombreuses, et ils ont plus d'enfants que les autres groupes sociaux, et le résultat est que les enfants risquent d'être plus pauvres que les adultes. Les ménages où la consommation ou le revenu par habitant sont faibles sont typiquement plus jeunes, et leurs membres sont plus susceptibles de mourir prématurément. Dans les pays en développement, la mortalité infantile et postinfantile pousse la population pauvrè des mariages précoces et à la prolificité pour parvenir à une famille complète. Les pauvres sont également incités à avoir uneforte fécondité pour le souci d'avoir des enfants qui les aident dans leur vieillesse, mais aussi la nécessité d'avoir de la main-d'oeuvre d'appoint dans le ménage ou dans l'exploitationagricole. Cesfacteurs tendent à abaisser la valeur moyenne du revenu par habitant, à faire obstacle à l'accumulation du capital physique et humain, et à entraîner une fragmentation des parcelles agricoles et donc un amenuisement des ressources foncières des pauvres, par rapport aux autres groupes sociaux. Toute stratégie de réduction de la pauvreté doit donc tenir compte de ces problemes démographiques ", ibid., p. 141.

(26) "Les difficultés rencontrées pour élaborer des directives à l'intention des gouvernements sont essentiellement dues à la diversité des valeurs, des normes, des besoins, des ressources, des priorités, des pratiques et des circonstances, qui entraîne elle-même une grande diversité de conceptions et de comportements, lesquels peuvent être rationnels, fonctionnels et appropriés dans un milieu et inadéquats dans un autre. Il semble qu'on ne puisse pas tirer de conclusions simples et universelles quant à ce qui serait bon ou mauvais pour les familles. Ainsi, une évolution peut être jugée positive dans un milieu culturel et négative dans un autre, ce qui peut être avantageux pour la 
personne peut ne pas l'être pour la famille. En outre ce qui est perçu comme un avantage à court terme peut poser des problèmes à long terme et vice versa ".

(27) De la sorte, le droit de fonder une famille est amplifié dans son sens libéral initial, le droit de choisir le nombre de ses enfants ce qui suscite des chapitres entiers extrêmement détaillés. Par exemple, dans le rapport de la Conférence du Caire sur la population le chapitre VII sur les droits et santé en matière de reproduction (sur lequel le Saint-Siège a encore émis une réserve) prône " un comportement sexuel et procréateur responsable ». Il part d'abord de l'idée d'espacement optimal des naissances dans la famille, pour surtout s'intéresser par le biais de l'information nécessaire aux familles, à la planification contraceptive, l'éducation sexuelle, la prévention du sida, la sexua- lité des adolescents, qui intègrent mal, tout en s'y référant, la responsabilité éducative des parents.

(28) Rapport du Comité pour l'élimination de la discrimination à l'égard des femmes, Document de la Conférence de Pékin, 21 juin 1995, A/Conf/177/7.

(29) Traite des femmes et des petites filles, point 109 de l'ordre du jour, Assemblée générale des NU, cinquantième session, A/50/369.

(30) Sur ces questions voir Katz C., Pour la proclamation par la communauté internationale d'un noyau intangible des droits del'homme, Revue trimestrielle des droits de l'homme, 1996, pp. 541-553. et Tavernier P., L'ONU et l'affirmation de l'universalité des droits de l'homme, Revue trimestrielle des droits de l'homme, 1997, pp. 385-393.

\section{Revue Recherches et Prévisions}

\section{Appel à articles}

La revue Recherches et Prévisions publie des articles concernant le champ familial et social : modes de vie, politiques publiques, acteurs et institutions.

Pour les modalités : voir dernière page du numéro. 